\title{
Coinheritance of Southeast Asian ovalocytosis and the $\beta$-thalassemia trait in a Malay family
}

\author{
Yamunah Raman ${ }^{\mathrm{a}}$, Mohamed Saleem ${ }^{\mathrm{a}}$, Rahimah Ahmad ${ }^{\mathrm{b}}$, Narazah Mohd Yusoff ${ }^{\mathrm{a}}$ \\ ${ }^{a}$ Advanced Medical and Dental Institute, Universiti Sains Malaysia, Pulau Pinang 13200, Malaysia \\ ${ }^{b}$ Institute for Medical Research, Kuala Lumpur, Wilayah Persekutuan 47000, Malaysia
}

\begin{abstract}
Background: Southeast Asian ovalocytosis (SAO) is a red blood cell membrane disorder caused by a 27 base pair (bp) deletion in the SLC4A1 that transcribes to a truncated variant of band-3 glycoprotein. $\beta$-Thalassemia is another red blood cell disorder, caused by mutant alleles in the $\beta$-globin gene that lead to globin chain impairment. Both conditions occur in Southeast Asian countries, including Malaysia.

Objectives: This report describes hematological and molecular features of a patient with both SAO and $\beta$-thalassemia and her children.

Methods: A 58-year-old Malay woman presented to our clinic with dizziness, tiredness, and easy fatigability. She was mildly anaemic. Analysis of her complete blood counts and her peripheral blood smear revealed microcytic hypochromic anaemia with large numbers of macro-ovalocytes and stomatocytes.

Results: Hemoglobin and globin gene studies revealed heterozygous $\beta$-thalassemia, and further analysis demonstrated a heterozygous 27 bp deletion on the SLC4A1 gene consistent with SAO. Analysis of the patient's offspring showed co-inheritance of $\beta$-thalassemia and SAO in her elder daughter and son, and SAO alone in another daughter.

Conclusions: High frequencies of SAO and different $\beta$-thalassemia mutations are present in the Malaysian population, thus coinheritance of SAO and $\beta$-thalassemia is not uncommon. However, this coinheritance is rarely reported, possibly because the red blood cell indices are overlooked and peripheral smear examinations are not routine.
\end{abstract}

Keywords: Malaysia, red blood cell membrane, Southeast Asian ovalocytosis, $\beta$-thalassemia trait

Southeast Asian ovalocytosis (SAO) is a red blood cell (RBC) membrane abnormality common in Southeast Asian populations [1, 2], including inhabitants of Papua New Guinea [3, 4]. In the Malaysian population, the prevalence of SAO is around $4 \%$ [5]. It is an asymptomatic hereditary autosomal dominant disorder characterized by macro-ovalocytes and stomatocytes constituting 25\% of the cells in peripheral blood smears (PBS). The underlying molecular abnormality is due to deletion of nine amino acids from the transmembrane protein called band-3, which has various structural and functional consequences $[6,7]$. $\beta$-Thalassemia is a RBC disorder caused by a mutant allele in the $\beta$-globin gene $(H B B)$ that leads to impaired synthesis of $\beta$-globin peptides [8]. $\beta$-Thalassemia is inherited as an autosomal recessive disorder and is characterized by

Correspondence to: Narazah Mohd Yusoff, Advanced Medical and Dental Institute, Universiti Sains Malaysia, Pulau Pinang 13200, Malaysia.E-mail: narazah@amdi.usm.edu.my hypochromic microcytic anaemia. This syndrome is common in Malaysia with an estimated carrier rate of $3.5 \%-4 \%$ and is identified as a public health problem. Despite the relatively high prevalence of these two disorders in Malaysia, coinheritance of SAO and the -thalassemia trait is rarely reported. Herein we describe the clinical presentation and laboratory variables for a series of patients from a single Malaysian Malay family with SAO and $\beta$ thalassemia.

\section{Case report}

This investigation was approved by the Human Ethics Committee, Universiti Sains Malaysia (approval ref. USMKK/PPP/JEPeM [245.3.(3)]). Written informed consent for publication of results was obtained from all examined family members.

A 58-year-old woman (K1) of Malay ancestry presented to the outpatient clinic of the Clinical Trial Centre, Advanced Medical and Dental Institute with 
shortness of breath, dizziness, and easy fatigability. She was mildly pale, and her physical examination was unremarkable with no evidence of visceral enlargement. Analysis of her complete blood counts using an XE-5000 hematology analyzer (Sysmex, Kobe, Japan) showed mild anemia for her age and sex, decreased mean corpuscular volume (MCV), and mean corpuscular hemoglobin (MCH), and elevated red cell distribution width $(\mathrm{RDW}=17.2)$. These features are suggestive of the presence of the thalassemia trait (Table 1). Her PBS examination revealed a dimorphic population of hypochromic microcytic red cells with a high number of macroovalocytes; moreover, transverse ridges and Y-shaped stroma were present in some of these cells, a presentation asynchronous for pure thalassemia. High performance liquid chromatography (HPLC; Variant, Bio-Rad Laboratories, Hercules, CA, USA) revealed a hallmark elevation of the hemoglobin $(\mathrm{Hb}) \mathrm{A}_{2}$ window to $5.0 \%$ and the $\mathrm{HbF}$ window to $1.5 \%$. Molecular analysis of $H B B$ revealed the presence of an IVS1-1 $(\mathrm{G}>\mathrm{T})$ mutation confirming the patient's status as heterozygous $\beta$-thalassemia. To determine the molecular basis of the macro-ovalocytes, we looked for the presence of a 27 base pair (bp) deletion in $S L C 4 A 1$, also known as B3 $\Delta 27$, that characterizes SAO. A set of Gap-PCR primers (5'-GGG CCC AGA TGA CCC TCT GC-3' and 5'-GCC GAA GGT GAT GGC GGG TG-3') that flanks the 27 bp deletion (NC_000017.11:g.44258044_44258070del27) on the tenth exon of SLC4A1 was used to amplify the region of interest [9]. The $148 \mathrm{bp}$ amplicon resolved on 2.5\% agarose gel demonstrated that SLC4A1 contained the pathognomonic $27 \mathrm{bp}$ deletion, which confirmed the presence of SAO in the patient. Subsequently, her two daughters and son were referred to the clinic for screening for the presence of SAO and the $\beta$-thalassemia trait.

The patient's eldest daughter (K2) was 35 years old and complained of easy fatigability. She was mildly anemic and had a dimorphic population of red cells, with hypochromic microcytic red cells, and a moderate degree of oval macrocytes. PBS also showed the presence of stomatocytes. HPLC analysis revealed the hallmark elevation of $\mathrm{HbA}_{2}$ (5.7\%), and polymerase chain reaction (PCR) analysis of $H B B$ and SLC4A1 identified the IVS1-1(G>T) mutation and 27 bp deletion respectively; these results confirmed that she inherited both the $\beta$-thalassemia trait and SAO.
The second daughter (K3) was 25 years old. Her blood counts and hemoglobin chromatography were unremarkable (Table 1). However, a significant number of oval macrocytes in a background of normocytic normochromic erythrocytes were present in her PBS. PCR analysis revealed the presence of the 27 bp deletion in SLC4A1, confirming the diagnosis of pure SAO.

The clinical and hematological presentation of the 20-year-old son (K4) was similar to that of his mother, the proband. Hemoglobin chromatography showed that $\mathrm{HbA}_{2}$ was $6.0 \%$, and $H B B$ and SLC4A1 characterization revealed a heterozygous IVS1-1 $(\mathrm{G}>\mathrm{T}$ ) mutation and $\mathrm{B} 3 \Delta 27$ confirming the coinheritance of $\beta$-thalassemia and SAO.

\section{Discussion}

Hereditary SAO is a genetic disorder characterized by the presence of large number of ovalocytes in the peripheral blood. This disorder is also referred to as hereditary ovalocytosis of Melanesians and stomatocytic elliptocytosis. SAO is frequent in Southeast Asia and Melanesia, occurring in up to $30 \%$ of people in some groups [1-4]. In Malaysia, the incidence of the disorder is estimated to be $4 \%$ [5]. Although the exact distribution in different ethnic groups in Malaysia is not known, Eng (1965) reported the incidence of hereditary ovalocytosis to be $12.3 \%$ in aborigines of the Senoi tribe of Malaysia [1].

Normal band-3 glycoproteins embedded in the bilipid layer of erythrocytes are multifunctional. They mediate anion transport across the membrane and respiration of carbon dioxide, and they provide a principle biding sites for ankyrin, a protein that anchors the cytoskeletal spectrin to the red cell membrane. The truncated variant of band-3 transmembrane protein (B3 $\Delta 27$ ) removes nine amino acids from residues 400 408 at the boundary between the cytoplasmic domain and the first transmembrane domain in RBCs [6, 7]. As a result, the band-3 glycoproteins in SAO remain tightly bound to ankyrin, reducing the lateral mobility of the membrane and affecting other membrane bound functions.

In general, individuals with a single copy of the variant $\mathrm{B} 3 \Delta 27$ deletion have asymptomatic ovalocytosis and do not require any form of treatment, whereas two copies is thought to be lethal in utero [9]. Relative to the poor genotype fitness in its homozygous state, heterozygotes are clinically asymptomatic and are protected against invasion by 


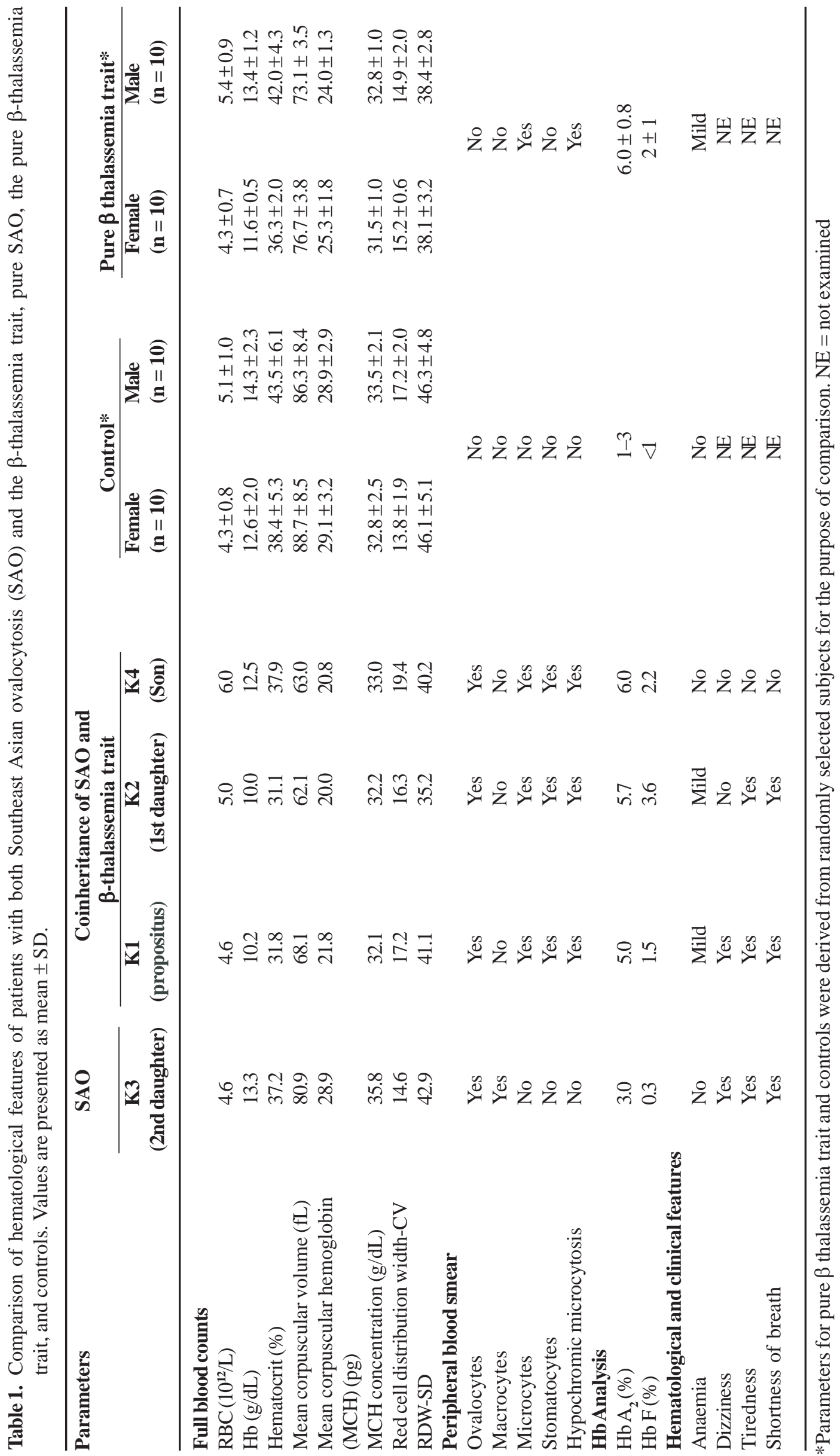


the malaria parasite because of the reduced deformability of red cells [10-12]. This overdominant selection makes the 27 bp deletion a balanced polymorphism allele. Both the $\beta$-globin gene and the $\mathrm{B} 3 \Delta 27$ deletion individually are thought to be maintained at high frequencies in malaria endemic regions by selection. When both alleles are coinherited in the same individual, as in $\mathrm{K} 1, \mathrm{~K} 2$, and $\mathrm{K} 4$, the protective effect against malaria is not well understood; however, we hypothesize that cells would remain more resistant because of the double mode of protection.

SAO transmitted as an autosomal dominant trait was reported previously in a Malaysian family [13], whereas a recessive pattern of inheritance was reported for a Melanesian family [14]; these conflicting results suggest that genetic entities differ between the two groups. The mode of inheritance in the family described herein could not be ascertained, because the father was not available for pedigree analysis. However, the finding that all of the patient's children had ovalocytosis with the same 27 bp deletion suggests dominant inheritance with a high level of penetrance within this family.

In patients with pure SAO, PBS is often pathognomonic, showing more than $25 \%$ of macroovalocytes with stomatocytes. These ovalocytes typically exhibit double Y- or V-shaped central pallors without associating polychromasia. The RDW and RBC histograms generated by automated blood cell counters may provide some useful information that is often beclouded to inexperienced laboratory workers. In a person with typical SAO, the RBC histogram is generally widened and has a bimodal distribution. A shoulder on the right side of the histogram represents oval macrocytes, whereas the dominant peak represents the second population of cells (either normocytic or microcytic erythrocytes).

Based on these three cases (K1, K2, and K4); the clinical expression of $\beta$ trait is not significantly aggravated by the coinheritance of SAO. The mild anaemia and reduced MCV and MCH observed in $\mathrm{K} 1$ and $\mathrm{K} 2$ reflect the primary pathophysiology of $\beta$-thalassemia, and not SAO. Although diagnosis of the $\beta$-thalassemia trait by measuring red cell indices and $\mathrm{HbA}_{2}$ is not influenced by the coinheritance of SAO, the blood smear examinations, and RDW and RBC histograms may provide clues to their coinheritance. In the $\mathrm{K} 1$ (proband), K2, and K4 patients in the present study, the RBC histogram had a bimodal pattern; the primary peak was shifted to the left, representing microcytic cells, because of the $\beta$-thalassemia trait, and the second shoulder on the right represented the oval-macrocytic population.

As shown in Table 1, the RDW-CV values observed for the controls compared with K1, K2, K3, and $\mathrm{K} 4$, despite having the same diagnosis, were less informative. This is probably because the measure RDW-CV represents a ratio of $\pm 1 S D$ to the mean MCV, and perhaps the oval macrocytes were not well represented within \pm 1 SD of the MCV. RDW$\mathrm{SD}$, therefore, should be a better index and a more accurate measure of erythrocyte anisocytosis, as it is a direct measure across the red cell size distribution and is independent of MCV. This index is particularly informative when a dimorphic red cell population is present, as in cases K1, K2, and K3 (represented by microcytes and macro-ovalocytes).

In some countries, single tube $(0.36 \% \mathrm{NaCl})$ osmotic fragility is used as a primary screening tool for $\beta$-thalassemia. However, the sensitivity of this test may be affected if the population has high segregation of SAO. For example, Fucharoen et al. showed that the hypotonic saline solution used for such screening produces a negative result when $\beta$-thalassemia is coinherited with SAO [15].

\section{Conclusion}

Because the frequencies of SAO and different $\beta$-thalassemia mutations are high in the Malaysian population, it is likely that coinheritance of the gene for SOA and the gene for $\beta$-thalassemia is not uncommon, although it is rarely reported.

\section{Acknowledgment}

We thank the staff of the Haematology Section, Advanced Diagnostic Laboratory, and Advanced Medical and Dental Institute of Universiti Sains Malaysia.

\section{Conflict of interest statement}

The authors have no conflicts of interest to declare.

\section{References}

1. Eng LIL Hereditary ovalocytosis and haemoglobin E-ovalocytosis in Malayan aborigines. Nature. 1965; 208:1329.

2. Takeshima Y, Sofro AS, Suryantoro P, Narita N, Matsuo M. Twenty seven nucleotide deletion within 
exon 11 of the erythrocyte band 3 gene in Indonesian ovalocytosis. Jpn J Hum Genet. 1994; 39:181-5.

3. Amato D, Booth PB. Hereditary ovalocytosis in Melanesians. P N G Med J. 1977; 48:102-8.

4. O’Donnell A, Allen SJ, Mgone CS, Martinson JJ, Clegg JB, Weatherall DJ. Red cell morphology and malaria anaemia in children with Southeast-Asian ovalocytosis band 3 in Papua New Guinea. Br J Haematol. 1998; 101: 407-12.

5. Yusoff NM, Van Rostenberghe H, Shirakawa T, Nishiyama K, Amin N, Darus Z, et al. High prevalence of Southeast Asian ovalocytosis in Malays with distal renal tubular acidosis. J Hum Genet. 2003; 48: 650-3.

6. Liu SC, Zhai S, Palek J, Golan DE, Amato D, Hassan K, et al. Molecular defect of the band 3 protein in southeast Asian ovalocytosis. N Engl J Med. 1990; 323:1530-8

7. Jarolim P, Palek J, Amato D, Hassan K, Sapak P, Nurse GT, et al. Deletion in erythrocyte band 3 gene in malaria-resistant Southeast Asian ovalocytosis. Proc Natl Acad Sci USA. 1991; 88:11022-6.

8. Weatherall DJ, Clegg JB. Inherited haemoglobin disorders: an increasing global health problem. Bull World Health Organ. 2001; 79:704-12.

9. Liu SC, Jarolim P, Rubin HL, Palek J, Amato D, Hassan K, et al. The homozygous state for the band
3 protein mutation in Southeast Asian Ovalocytosis may be lethal. Blood. 1994; 84:3590-1. [Letter]

10. Cattani JA, Gibson FD, Alpers MP, Crane GG. Hereditary ovalocytosis and reduced susceptibility to malaria in Papua New Guinea. Trans R Soc Trop Med Hyg. 1987; 81:705-9.

11. Allen SJ, O’Donnell A, Alexander ND, Mgone CS, Peto TE, Clegg JB, et al. Prevention of cerebral malaria in children in Papua New Guinea by southeast Asian ovalocytosis band 3. Am J Trop Med Hyg. 1999; 60:1056-60.

12. Mohandas N, Lie-Injo LE, Friedman M, Mak JW. Rigid membranes of Malayan ovalocytes: a likely genetic barrier against malaria. Blood. 1984; 63: 1385-92.

13. Fix AG, Baer AS, Lie-Injo LE. The mode of inheritance of ovalocytosis/elliptocytosis in Malaysian Orang Asli families. Hum Genet. 1982; 61:250-3.

14. Kimura M, Tamam M, Soemantri A, Nakazawa M, Ataka Y, Ohtsuka R, et al. Distribution of a 27-bp deletion in the band 3 gene in South Pacific islanders. J Hum Genet. 2003; 48:642-5.

15. Fucharoen G, Fucharoen S, Singsanan S, Sanchaisuriya K. Coexistence of Southeast Asian ovalocytosis and beta-thalassemia: a molecular and hematological analysis. Am J Hematol. 2007; 82:381-5. 